\title{
High-Order Spectral Finite Elements in Analysis of Collinear Wave Mixing
}

\author{
Changfa Ai, ${ }^{1,2}$ Youxuan Zhao, ${ }^{3}$ Peng Cao, ${ }^{4}$ Enhui Yang, ${ }^{1,2}$ and Yanjun Qiu ${ }^{1,2}$ \\ ${ }^{1}$ School of Civil Engineering, Southwest Jiaotong University, Chengdu, Sichuan 610031, China \\ ${ }^{2}$ Key Laboratory of Highway Engineering of Sichuan Province, Southwest Jiaotong University, Chengdu, Sichuan 610031, China \\ ${ }^{3}$ College of Aerospace Engineering, Chongqing University, Chongqing 400044, China \\ ${ }^{4}$ Department of Hydraulic Engineering, Tsinghua University, Beijing 100084, China
}

Correspondence should be addressed to Youxuan Zhao; youxuan.zhao@gmail.com

Received 8 October 2015; Revised 25 November 2015; Accepted 26 November 2015

Academic Editor: Roman Lewandowski

Copyright (C) 2015 Changfa Ai et al. This is an open access article distributed under the Creative Commons Attribution License, which permits unrestricted use, distribution, and reproduction in any medium, provided the original work is properly cited.

\begin{abstract}
Implementing collinear wave mixing techniques with numerical methods to detect acoustic nonlinearity due to damage and defects is of vital importance in nondestructive examination engineering. However, numerical simulations in existing literatures are often limited due to the compromise between computational efficiency and accuracy. In order to balance the contradiction, spectral finite element (abbreviated as SFE) with $3 \times 3$ and $8 \times 6$ nodes is developed to simulate collinear wave mixing for $1 \mathrm{D}$ and $2 \mathrm{D}$ cases in this study. The comparisons among analytical solutions, experiments, finite element method (FEM), and spectral finite element method are presented to validate the feasibility, efficiency, and accuracy of the proposed SFEs. The results demonstrate that the proposed SFEs are capable of increasing computational efficiency by as much as 14 times while maintaining the same accuracy in comparison with FEM. In addition, five $3 \times 3$ nodes' SFEs or one $8 \times 6$ nodes' SFE per the shortest wavelength is sufficient to capture mixing waves. Finally, the proposed $8 \times 6$ nodes' SFE is recommended for collinear wave mixing to detect damage, which can offer more accuracy with similar efficiency compared to $3 \times 3$ nodes' SFE.
\end{abstract}

\section{Introduction}

Recently, wave mixing techniques with noncollinear $[1,2]$ and collinear [3-6] incident waves have been used to detect the change of material nonlinearity caused by plasticity and fatigue damage. The techniques are less sensitive to the measurement system nonlinearity and can detect the nonlinearity of the mixing zone instead of the average value during incident wave propagation. The nonlinear wave mixing techniques have some unique advantages compared to traditional nonlinear wave techniques. For instance, frequencies can be selected according to the requirements of users, which can avoid unwanted harmonics typically generated by a number of electronic components in the measurement system. Besides, researchers can scan over the regions of interest directly by controlling the wave mixing locations.

Collinear wave mixing techniques were studied analytically, numerically, and experimentally by Tang et al. [3$5,7]$ and Chen et al. [6,7]. Chen et al. [6] derived a set of necessary and sufficient conditions for generating resonant waves by two propagating time-harmonic plane waves and obtained closed-form analytical solutions to resonant waves generated by two collinearly propagating sinusoidal pulses. Numerical simulations based on finite element method and experimental measurements using one-way mixing were conducted. However, waveforms and propagation rules of mixing waves can be obtained from the analytical solutions [6] only for plane wave (1D) case in the semi-infinite domain when resonant conditions are strictly satisfied. In realistic experimental measurements, the limitations, such as frequency deviation due to inaccurate acoustic velocity of material or quasi-collinear wave generation, could result in imperfect resonant conditions when using collinear wave mixing techniques. Therefore, it is necessary to simulate collinear wave mixing with numerical methods, which are the extension and supplement to the analytical solutions and the experiments.

For the past two decades, various numerical methods including finite difference method $[8,9]$, finite element 
method [10, 11], boundary element method [12, 13], finite strip element method $[14,15]$, mass-spring lattice model $[16,17]$, and local interaction simulation approach $[18,19]$ have been applied to simulate wave propagation. Finite element method requires strict rules for spatial and temporal discretization to study the interaction of waves, which can cause numerical problems in the cases of high frequencies and great dimensions [20-22]. No less than 20 first-order 4node reduced elements per wavelength of the highest frequencies are required to capture nonlinear interaction [23]. Therefore, finite element method is limited due to the contradiction between accuracy and efficiency for high frequency wave propagation. However, the orthogonal polynomialsbased spectral finite element method [24-26] is much more suitable for analyzing wave propagation in structures with complex geometry. This method is characterized by highorder orthogonal polynomials as approximation functions with diagonal mass matrix obtained naturally. More recently, spectral finite element method was used to simulate wave propagation in structures. Wave propagation in $1 \mathrm{D}$ structures, such as rod and beam, was investigated by some researches [27-31]. Numerical simulations of transverse wave propagation in a composite plate were presented by Kudela et al. [32]. Komatitsch et al. [33] applied a spectral element method based upon a conforming mesh of quadrangles and triangles to the problem of $2 \mathrm{D}$ elastic wave propagation. Rekatsinas et al. [34] developed a time-domain spectral finite element for improving the efficiency of numerical simulations of guided waves in laminated composite strips. The applications of $3 \mathrm{D}$ spectral finite element to wave propagation problems [35-39] were also investigated in many fields.

However, the applications of spectral finite element method for wave mixing techniques have not been widely reported in literatures so far. In this paper, two types of spectral finite elements are developed to simulate collinear wave mixing for damage detection. Results from analytical solutions, experiments, FEM, and SFE are compared to validate the feasibility, efficiency, and accuracy of the proposed SFEs. Finally, the comparison between two types of spectral finite elements is investigated by considering the contradiction between accuracy and efficiency.

\section{Theory for Collinear Mixing of Wave Pulses}

Consider a homogeneous solid with quadratic nonlinearity; the displacement equations of motion in one dimension can be written as [6]

$$
\begin{aligned}
& \frac{\partial^{2} u_{1}}{\partial t^{2}}-c_{L}^{2} \frac{\partial^{2} u_{1}}{\partial x^{2}}=\beta_{L} c_{L}^{2} \frac{\partial u_{1}}{\partial x} \frac{\partial^{2} u_{1}}{\partial x^{2}}+\beta_{T} c_{T}^{2} \frac{\partial u_{2}}{\partial x} \frac{\partial^{2} u_{2}}{\partial x^{2}}, \\
& \frac{\partial^{2} u_{2}}{\partial t^{2}}-c_{T}^{2} \frac{\partial^{2} u_{2}}{\partial x^{2}}=\beta_{T} c_{T}^{2}\left(\frac{\partial u_{1}}{\partial x} \frac{\partial^{2} u_{2}}{\partial x^{2}}+\frac{\partial u_{2}}{\partial x} \frac{\partial^{2} u_{1}}{\partial x^{2}}\right),
\end{aligned}
$$

where $u_{1}$ and $u_{2}$ are the two components of the displacement and $c_{L}=\sqrt{(\lambda+2 \mu) / \rho}$ and $c_{T}=\sqrt{\mu / \rho}$ are the longitudinal and transverse phase velocities, respectively. $\beta_{L}=3+$ $2(l+2 m / \lambda+2 \mu)$ and $\beta_{T}=(\lambda+2 \mu) / \mu+m / \mu$ are called, respectively, the longitudinal and transverse acoustic nonlinearity parameters. $\lambda$ and $\mu$ are the Lamé constants, $\rho$ is the mass density, and $l, m$, and $n$ are the Murnaghan thirdorder elastic constants.

When a longitudinal wave pulse and a transverse wave pulse are both emitted at $x=0$ and propagate in the positive $x$-direction, it is called one-way mixing. When the transverse pulse is emitted at $x=0$ and propagates in the positive $x$ direction, while the longitudinal pulse is emitted at $x=L$ and propagates in the negative $x$-direction, it is called twoway mixing. A resonant transverse wave will be generated and propagate in the opposite direction of the primary transverse wave, if resonance conditions $\omega_{L} / \omega_{T}=2 \kappa /(\kappa+1)$ for one-way mixing and $\omega_{L} / \omega_{T}=2 \kappa /(\kappa-1)$ for two-way mixing can be satisfied, respectively. The frequencies of resonant waves for both cases are $\omega_{R}=\omega_{L}-\omega_{T}$, where $\kappa=c_{L} / c_{T}$ and $\omega_{T}$ and $\omega_{L}$ are the circular frequencies of the longitudinal and transverse waves, respectively.

The signal received at $x=0$ for one-way mixing can be expressed as

$$
u_{2}(0, t)=\frac{\beta_{T} \omega_{T}^{2} U V}{2 c_{T}^{2}(\kappa+1)}\left(\frac{c_{L} \tau_{L}}{\kappa+1}\right) g\left(t, n_{L}, \phi_{-}\right) .
$$

The signal received at $x=0$ for two-way mixing can be expressed as

$$
u_{2}(0, t)=\frac{\beta_{T} \omega_{T}^{2} U V}{2 c_{T}^{2}(\kappa-1)}\left(\frac{c_{T} \tau_{T}}{2}\right) g\left(t, n_{T},-\phi_{-}\right) .
$$

The detailed expressions of (2) and (3) are given in [6].

\section{Formulation of 2D Spectral Finite Element}

The spectral finite element formulation [27, 33, 39] process of the stiffness and mass matrices is similar to the traditional finite element formulation. The domain $\Omega$ in $2 \mathrm{D}$ is firstly meshed to a number of nonoverlapping quadrilaterals. The quadrilateral spectral finite element defined on the domain $\Omega_{e}$ is subsequently mapped from the physical coordinate $(x, y)$ to the reference domain $\Lambda$ with $(\xi, \eta) \in[-1,1] \times[-1,1]$ using invertible local mapping $\mathscr{F}_{e}: \Lambda \rightarrow \Omega_{e}$. In the reference domain $\Lambda$, a set of local shape functions are defined consisting of Lagrange polynomials of degree $N$. The local nodes $\xi_{i} \in[-1,1], i \in 1, \ldots,(N+1)$, are defined as GaussLobatto-Legendre (GLL) points which are $(N+1)$ roots of the equation

$$
\left(1-\xi^{2}\right) P_{N}^{\prime}(\xi)=0
$$

where $P_{N}^{\prime}(\xi)$ denotes the first derivative of the Legendre polynomial of degree $N$.

The displacement field within the quadrangular element can be approximated as

$$
u_{N}^{e}(\xi, \eta)=\sum_{i=1}^{N+1} \sum_{j=1}^{N+1} q_{N}^{e}\left(\xi_{i}, \eta_{j}\right) \psi_{i}(\xi) \psi_{j}(\eta),
$$

where $q_{N}^{e}\left(\xi_{i}, \eta_{j}\right)$ denotes nodal degrees of freedom and $\psi_{i}\left(\xi_{j}\right)$ denotes the $i$ th Lagrange interpolation at $(N+1)$ GLL points 
$\xi_{j} \cdot \psi_{i}\left(\xi_{j}\right)$ is equal to 1 at $j=i$ and 0 at all other points $j \neq i$. From this definition, a fundamental property appears, $\psi_{i}\left(\xi_{j}\right)=\delta_{i j}$, where $\delta_{i j}$ denotes the Kronecker delta.

Similar to the traditional finite element method, the element matrices $\mathbf{M}^{e}$ and $\mathbf{K}^{e}$ and the vector $\mathbf{F}^{e}$ are formulated numerically as follows:

$$
\begin{aligned}
\mathbf{M}^{e} & =\int_{\Omega_{e}} \rho\left(\psi^{e}(x, y)\right)^{T} \psi^{e}(x, y) \mathrm{d} x \mathrm{~d} y \\
& \approx \rho \sum_{i=1}^{N+1} \sum_{j=1}^{N+1} w_{i} w_{j}\left(\psi^{e}\left(\xi_{i}\right) \psi^{e}\left(\eta_{j}\right)\right)^{T} \\
& \cdot\left(\psi^{e}\left(\xi_{i}\right) \psi^{e}\left(\eta_{j}\right)\right) \operatorname{det}\left(\mathbf{J}^{e}\left(\xi_{i}, \eta_{j}\right)\right), \\
\mathbf{K}^{e} & =\int_{\Omega_{e}}\left(\mathbf{B}^{e}(x, y)\right)^{T} \mathbf{D}^{e} \mathbf{B}^{e}(x, y) \mathrm{d} x \mathrm{~d} y \\
& \approx \sum_{i=1}^{N+1} \sum_{j=1}^{N+1} w_{i} w_{j}\left(\mathbf{B}^{e}\left(\xi_{i}, \eta_{j}\right)\right)^{T} \mathbf{D}_{i j}^{e} \mathbf{B}^{e}\left(\xi_{i}, \eta_{j}\right) \\
& \cdot \operatorname{det}\left(\mathbf{J}^{e}\left(\xi_{i}, \eta_{j}\right)\right), \\
\mathbf{F}^{e}= & \int_{\Omega_{e}}\left(\psi^{e}(x, y)\right)^{T} \mathbf{p}(x, y)^{e} \mathrm{~d} x \mathrm{~d} y \\
& \approx \sum_{i=1}^{N+1} \sum_{j=1}^{N+1} w_{i} w_{j}\left(\psi^{e}\left(\xi_{i}\right) \psi^{e}\left(\eta_{j}\right)\right)^{T} \mathbf{p}\left(\xi_{i}, \eta_{j}\right)^{e} \\
\cdot & \operatorname{det}\left(\mathbf{J}^{e}\left(\xi_{i}, \eta_{j}\right)\right),
\end{aligned}
$$

where $\mathbf{D}^{e}$ is termed the material stiffness matrix, $\mathbf{p}(x, y)^{e}$ is a distributed load, and $\mathbf{J}^{e}$ is the Jacobian associated with the mapping $\mathscr{F}_{e}$ from the element $\Omega_{e}$ to the reference domain. The superscript $e$ represents the matrix of the element in the local coordinates both in linear and in nonlinear conditions.

The quadratic nonlinear elastic constitutive relation [40] including second-order constants and third-order constants is used, which is expressed using Voigt's notation $C_{i j k l}=c_{I J}$, $C_{i j k l m n}=c_{I J K}$ :

$$
\begin{aligned}
\sigma_{i j}= & C_{i j k l} E_{k l}+\frac{1}{2} C_{i j k l m n} E_{k l} E_{m n}, \\
C_{i j k l}= & \lambda \delta_{i j} \delta_{k l}+\mu\left(\delta_{i k} \delta_{j l}+\delta_{i l} \delta_{j k}\right), \\
C_{i j k l m n} & \\
= & (2 l-2 m+n) \delta_{i j} \delta_{k l} \delta_{m n} \\
& +(2 m-n)\left(\delta_{i j} I_{k l m n}+\delta_{k l} I_{m n i j}+\delta_{m n} I_{i j k l}\right) \\
& +\frac{n}{2}\left(\delta_{i k} I_{j l m n}+\delta_{i l} I_{j k m n}+\delta_{j k} I_{i l m n}+\delta_{j l} I_{i k m n}\right),
\end{aligned}
$$

where $E$ is the Lagrangian or Green strain, $I, J, K \in$ $\{1,2,3,4,5,6\}, i j=11,22,33,23,31,12 \leftrightarrow I=1,2,3,4,5,6$.

The matrix $\mathbf{B}^{e}$ is connected with approximated strains:

$$
\mathbf{B}^{e}=\mathbf{L} \psi^{e}\left(\xi_{i}\right) \psi^{e}\left(\eta_{j}\right)
$$

where $\mathbf{L}$ denotes a differential operator matrix

$$
\begin{aligned}
& \mathbf{L}=\left[\begin{array}{cc}
\frac{\partial}{\partial x} & 0 \\
0 & \frac{\partial}{\partial y} \\
\frac{\partial}{\partial y} & \frac{\partial}{\partial x}
\end{array}\right] \\
& {\left[\begin{array}{c}
\frac{\partial}{\partial x} \\
\frac{\partial}{\partial y}
\end{array}\right]=\mathbf{J}^{e}\left[\begin{array}{c}
\frac{\partial}{\partial \xi} \\
\frac{\partial}{\partial \eta}
\end{array}\right] \text {. }}
\end{aligned}
$$

The quadrature weights $w_{i}$, which are independent of the element, are determined by

$$
w_{i}=\frac{2}{n(n-1)\left[P_{n-1}\left(\xi_{i}\right)\right]^{2}}, \quad i \in 1, \ldots, n, n=N+1 .
$$

The element matrices are assembled to the global coordinate system and finally a modeling problem of wave propagation is reduced to a well-known ordinary differential equation, which can be written as

$$
\mathbf{M}(U) \ddot{\mathbf{U}}+\mathbf{C}(U) \dot{\mathbf{U}}+\mathbf{K}(U) \mathbf{U}=\mathbf{F},
$$

where $\mathbf{M}(U)$ is the global mass matrix, $\mathbf{C}(U)$ is the global damping matrix, and $\mathbf{K}(U)$ is the global stiffness matrix. They are all the functions of displacements $U$ in the nonlinear conditions. $\mathbf{F}$ is a vector of the time-dependent excitation signal.

Because of the excellent property of the SFE with the diagonal mass matrix, it is especially suitable for explicit scheme (central difference method) to discretize the secondorder ordinary differential equation in time. Therefore, based on the finite element method software ABAQUS, two types of $2 \mathrm{D}$ spectral finite elements $(3 \times 3$ and $8 \times 6$ nodes $)$ are developed via the user defined element subroutine of explicit solver (VUEL) using FORTRAN language to simulate collinear wave mixing. The scheme of the spectral finite element with $3 \times 3$ nodes for 2 -order Legendre polynomial both in $\xi$ and in $\eta$ direction is shown in Figure 1(a). Similarly, the spectral finite elements with $8 \times 6$ nodes for 7 -order Legendre polynomial in $\xi$ direction and 5-order Legendre polynomial in $\eta$ direction are shown in Figure 1(b). The flowchart of VUEL subroutine is listed in Figure 2.

\section{Numerical Simulations}

In this section, the feasibility of using spectral finite element method to simulate collinear wave mixing is investigated. The results of $1 \mathrm{D}$ case from the SFEs illustrated in Section 3 are compared with analytical and FEM results. It should be mentioned that the 4-node quadrilateral elements are utilized in the various condition for providing the comparison. The 2D half-space results from the SFEs are also compared with experimental and FEM results. The purpose is to determine the feasibility, efficiency, and accuracy in SFE simulations 


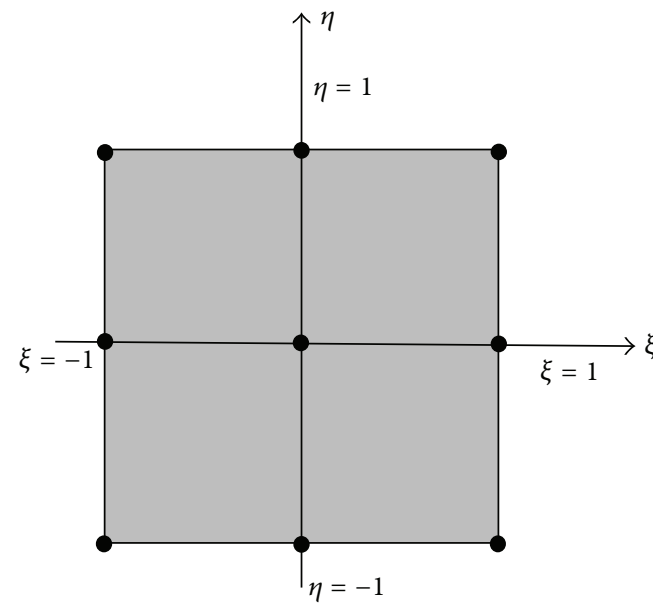

(a) A $3 \times 3$ nodes' spectral finite element

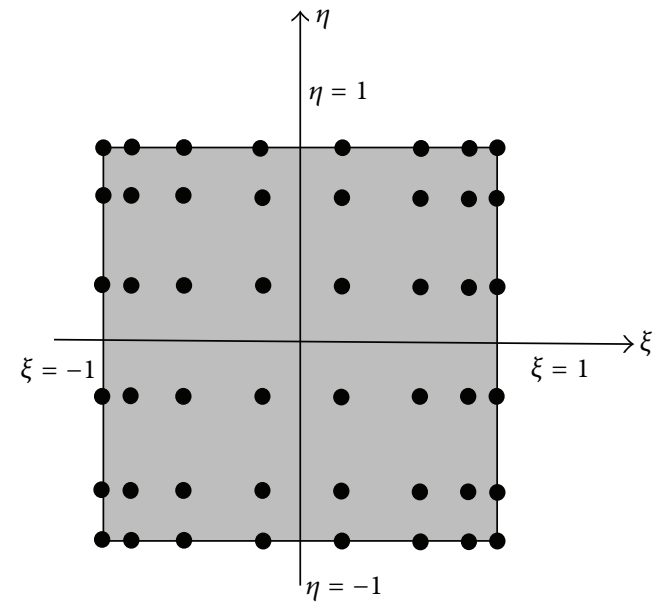

(b) An $8 \times 6$ nodes' spectral finite element

FIGURE 1: The schemes of spectral finite elements developed in this paper.

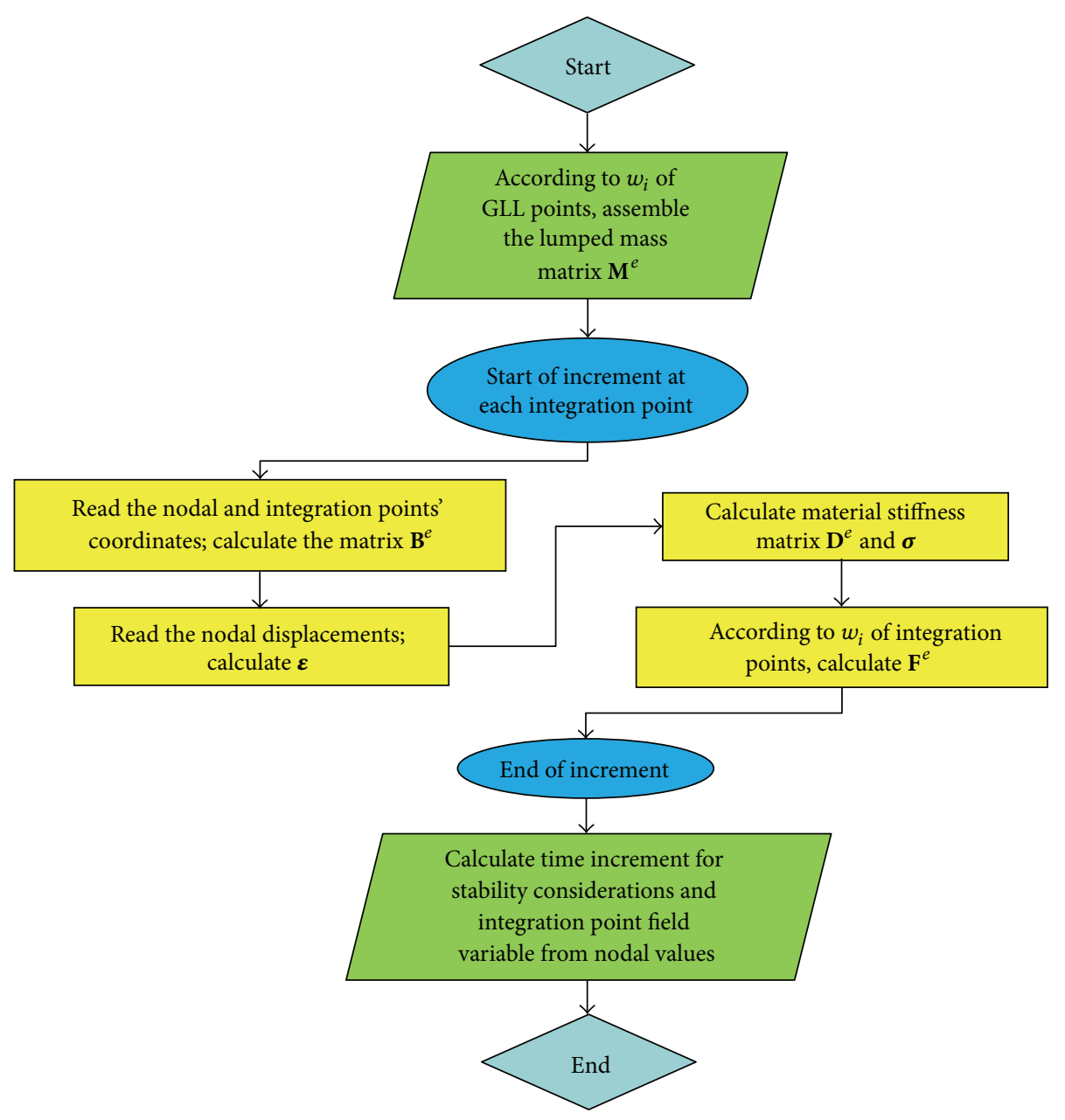

FIGURE 2: Flowchart of VUEL subroutine. 
to capture resonant waves. In SFE simulations, aluminum is used with the mechanical parameters [41] as follows: Young's modulus is $70 \mathrm{GPa}$, Poisson ratio is 0.33 , mass density is $2700 \mathrm{~kg} / \mathrm{m}^{3}$, and Murnaghan third-order elastic constants are $l=-126 \mathrm{GPa}, m=-320 \mathrm{GPa}$, and $n=-282 \mathrm{GPa}$.

4.1. Plane Wave Simulations. To simulate plane waves, a rectangular strip of $100 \mathrm{~mm} \times 5 \mathrm{~mm}$ is used in SFE-based model. The model consists of $62,5003 \times 3$ nodes' SFEs with 505,202 DOFs. A longitudinal and transverse wave pulse are generated from either the same end (one-way mixing) or opposite ends (two-way mixing). The source to generate these two pulses is uniformly distributed over the entire end surface of the rectangular strip, and periodic boundary conditions are used on the top and bottom surfaces of the rectangular strip. The receiver is located at the same position as the source of the transverse wave. The transverse pulse contains 10 cycles with the amplitude of $10^{-4} \mathrm{~mm}$, and the longitudinal pulse contains 5 cycles with the amplitude of $10^{-5} \mathrm{~mm}$.

For one-way mixing, the frequencies used are $\omega_{T}=$ $7.5 \mathrm{MHz}$ for the transverse wave and $\omega_{L}=10 \mathrm{MHz}$ for the longitudinal wave, which satisfy the resonant condition $\omega_{L} / \omega_{T}=2 \kappa /(\kappa+1)$. The corresponding resonant frequency is $\omega_{R}=2.5 \mathrm{MHz}$. In the case of two-way mixing, the frequencies used are $\omega_{T}=2.5 \mathrm{MHz}$ for the transverse wave and $\omega_{L}=$ $10 \mathrm{MHz}$ for the longitudinal wave, which satisfy the resonant condition $\omega_{L} / \omega_{T}=2 \kappa /(\kappa-1)$. The corresponding resonant frequency is $\omega_{R}=7.5 \mathrm{MHz}$.

It is estimated that the shortest wavelength is about 20 times the largest element size for FEM simulations with 4node quadrilateral elements. In SFE simulations with $3 \times 3$ nodes' SFEs, five elements per the shortest wavelength are sufficient to capture resonant waves. Time increment $\Delta t=$ $2.5 \times 10^{-10} \mathrm{sec}$ is conservatively conducted for explicit scheme. SFE simulations are conducted with 12 cores in parallel on Quest high performance computing cluster at Northwestern University.

The analytical and FEM results are also conducted to validate the feasibility, efficiency, and accuracy of the proposed SFEs. Shown in Figures 3(a) and 3(b) with blue dashed lines are the time-domain waveforms of resonant waves generated by one-way and two-way mixing using $3 \times 3$ nodes' SFE, respectively. The computation time and error of $3 \times 3$ nodes' SFE are listed in Table 1 in comparison with FEM. The excellent comparison shows that the proposed $3 \times 3$ nodes' SFE is capable of capturing accurately mixing waves. Five $3 \times 3$ nodes' SFEs per the shortest wavelength can provide sufficient accuracy compared with analytical solutions. Further, the 3 $\times 3$ nodes' SFE reduces two-thirds of DOFs and CPU time compared with FEM. It is shown that the $3 \times 3$ nodes' SFE can provide more efficiency with sufficient accuracy than FEM. In other words, when FEM could not deal with the cases of higher frequencies and greater dimensions, SFE could be a viable numerical method for those cases.

The proposed $8 \times 6$ nodes' SFE is also employed for oneway and two-way mixing cases. The model is meshed to 2,500 $8 \times 6$ nodes' SFEs with 178,602 DOFs. Because of the higher precision Legendre polynomial in $8 \times 6$ nodes' SFE, only one element per the shortest wavelength is sufficient to capture
TABLE 1: The comparison between SFEs and FEM for plane wave case.

\begin{tabular}{lccc}
\hline & $3 \times 3$ nodes' SFE & $8 \times 6$ nodes' SFE & FEM \\
\hline DOFs & 505,202 & 178,602 & $1,608,402$ \\
Error & $1.85 \%$ & $0.15 \%$ & $2.27 \%$ \\
CPU time & $3 \mathrm{~h} 15 \mathrm{~min}$ & $4 \mathrm{~h} 30 \mathrm{~min}$ & $10 \mathrm{~h}$ \\
\hline
\end{tabular}

resonant waves. The results of $8 \times 6$ nodes' SFE are compared with analytical, FEM, and $3 \times 3$ nodes' SFE, which are shown in Figure 3 and Table 1 . The comparison shows that the $8 \times 6$ nodes' SFE provides more accuracy than both FEM and $3 \times 3$ nodes' SFE do. Although CPU time for the simulation using 8 $\times 6$ nodes' SFE is marginally more than $3 \times 3$ nodes' SFE, the $8 \times 6$ nodes' SFE still reduces $55 \%$ of that CPU time compared with FEM.

4.2. 2D Half-Space Simulations. Ultrasonic tests are usually conducted on finite-size samples, and the pulses are generated by transducers of finite aperture as well. Thus, it is necessary to simulate the actual wave fields generated by finite-size transducers in finite-size samples using numerical simulations. All the physical processes of generating resonant waves are the same in $2 \mathrm{D}$ and $3 \mathrm{D}$ simulations. However, 3D simulations may cause super-large-scale computation up to one hundred million DOFs. Due to the limitation of computing resource, $2 \mathrm{D}$ half-space simulations are employed to simulate one-way and two-way mixing compared with experimental measurements.

The 2D SFE model is a $150 \mathrm{~mm} \times 144 \mathrm{~mm}$ rectangular strip with 450,000 $3 \times 3$ nodes' SFEs and 5,000 plane strain infinite elements (CINPE4). CINPE4 element can introduce nonreflecting boundary conditions to simulate the halfspace case. The frequencies and amplitudes of transverse and longitudinal wave pulses are the same as those in plane wave simulations for one-way and two-way mixing. The transverse and longitudinal pulses both contain 10 cycles. However, the Gaussian distribution $\left(f(r)=E_{0} \exp \left(-r^{2} / \omega_{0}^{2}\right)\right)$ in $x-y$ plane is used for the transverse and longitudinal waves, which is different from plane wave simulations. For oneway mixing, $6 \mathrm{~mm}$ surface above the original point at the $x$-axis end of sample is used to generate transverse wave pluses and receive mixing wave signals, while another $6 \mathrm{~mm}$ surface below the original point at the same end is used to generate longitudinal wave pluses. And in the case of twoway mixing, $12 \mathrm{~mm}$ surface at the $x$-axis end of sample is used to generate transverse wave pluses and receive mixing wave signals, while $12 \mathrm{~mm}$ surface at the opposite end is used to generate longitudinal wave pluses.

The model is also meshed to $33,5008 \times 6$ nodes' SFEs. The SFEs simulations are conducted with 144 cores via distributed parallel computation. The time-domain waveforms of resonant waves generated by one-way and two-way mixing using $8 \times 6$ nodes' SFE are shown in Figures 4(a) and 4(b), respectively. The experimental results of one-way mixing [6] and two-way mixing [3] are included to compare with 2D SFE simulations. Excellent agreement between SFE results and experimental measurements is observed. This demonstrates 


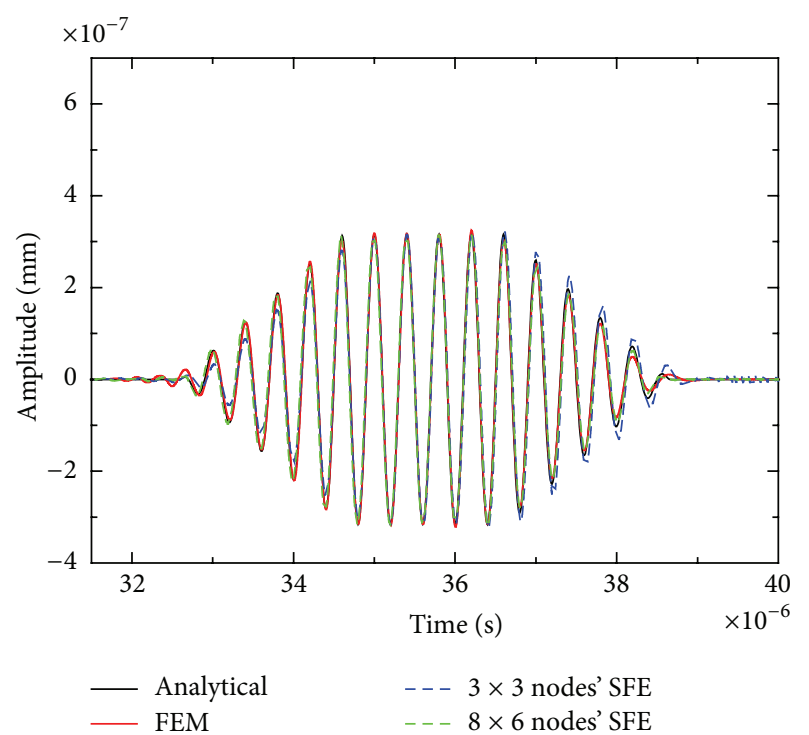

(a)

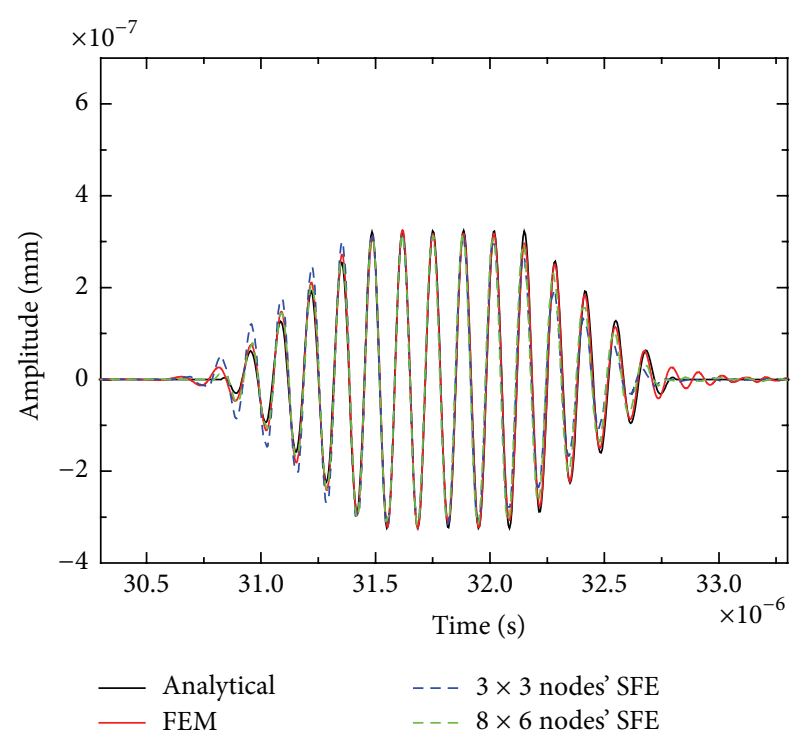

(b)

FIGURE 3: Waveforms of resonant waves generated by the one-way (a) and two-way (b) mixing.

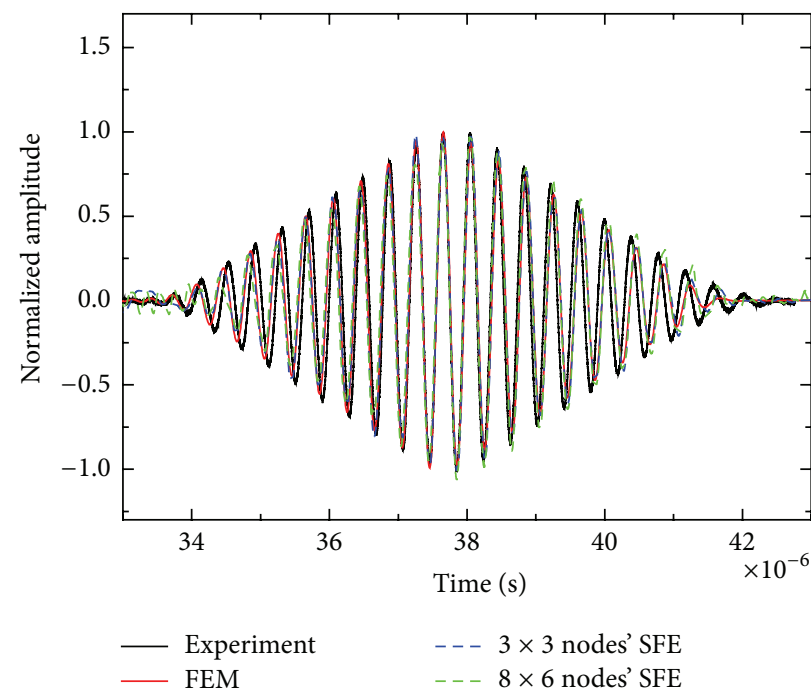

(a)

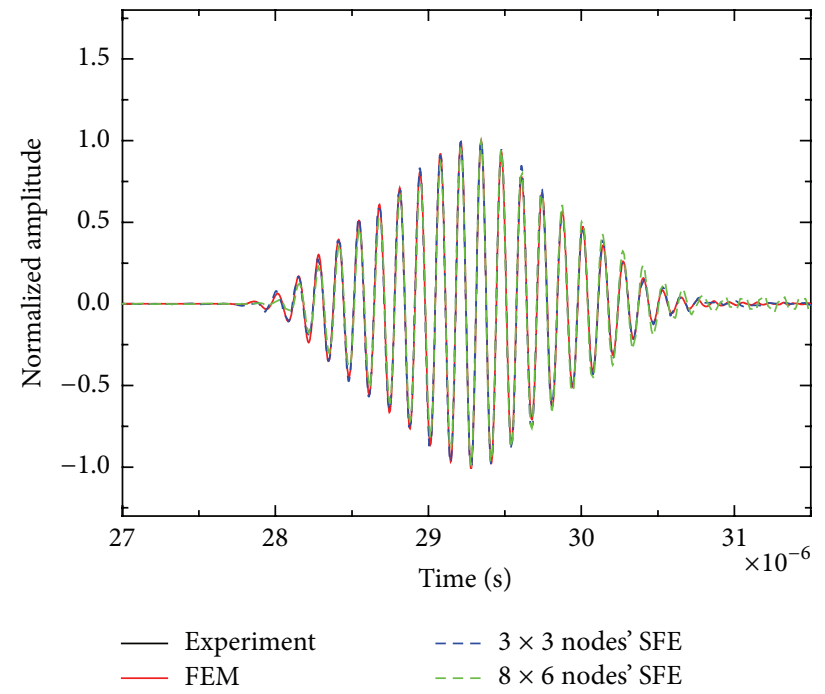

(b)

Figure 4: Waveforms of resonant waves from the one-way (a) and two-way (b) mixing for 2D case.

that the proposed SFEs could be viable numerical methods to simulate collinear wave mixing for damage detection. The computation times of SFEs are listed in Table 2 in comparison with FEM. The SFEs are capable of increasing computational efficiency by as much as 14 times compared to FEM.

In FEM simulations, 20 first-order elements per wavelength are required to precisely capture every waveform propagating in the medium, which leads to significant largescale computations for high frequency waves. However, due to the high precision Legendre polynomial in spectral finite element, only five $3 \times 3$ nodes' SFEs or one $8 \times 6$ nodes' SFE per wavelength is sufficient to accurately simulate wave propagation. Therefore, spectral finite element method can reduce computing scale and improve efficiency dramatically.
Both proposed $3 \times 3$ and $8 \times 6$ nodes' SFE can provide sufficient accuracy and excellent efficiency compared to FEM. By contrast, the $8 \times 6$ nodes' SFE can provide more accuracy with similar efficiency. Therefore, it is recommended that the proposed $8 \times 6$ nodes' SFE is suitable to capture resonant waves from collinear wave mixing.

\section{Summary and Conclusions}

In this paper, $3 \times 3$ and $8 \times 6$ nodes' spectral finite elements for nonlinear wave are developed. A series of collinear wave mixing simulations for $1 \mathrm{D}$ and $2 \mathrm{D}$ cases with the proposed spectral finite elements are conducted in this study. The feasibility, efficiency, and accuracy of these spectral finite elements 
TABLE 2: The comparison between SFEs and FEM for 2D simulations.

\begin{tabular}{|c|c|c|c|c|c|c|}
\hline & \multicolumn{3}{|c|}{ One-way mixing } & \multicolumn{3}{|c|}{ Two-way mixing } \\
\hline & $3 \times 3$ nodes' SFE & $8 \times 6$ nodes' SFE & FEM & $3 \times 3$ nodes' SFE & $8 \times 6$ nodes' SFE & FEM \\
\hline DOFs & $4,516,806$ & $2,111,706$ & $15,878,646$ & $4,516,806$ & $2,111,706$ & $15,878,646$ \\
\hline CPU time & $0 \mathrm{~h} 20 \mathrm{~min}$ & $0 \mathrm{~h} 24 \mathrm{~min}$ & $2 \mathrm{~h} 30 \mathrm{~min}$ & $0 \mathrm{~h} 15 \mathrm{~min}$ & $0 \mathrm{~h} 20 \mathrm{~min}$ & $3 \mathrm{~h} 30 \mathrm{~min}$ \\
\hline
\end{tabular}

are validated in comparison with analytical solutions, FEM, and experiments.

The present work shows that the proposed spectral finite elements provide efficient tools to simulate collinear wave mixing for damage detection. The advantages of these spectral finite elements can be summarized as follows:

(1) Due to the high precision Legendre polynomial, the SFEs-based model can significantly reduce the number of elements per wavelength to capture mixing waveforms compared with the FEM-based model. Only five $3 \times 3$ nodes' SFEs or one $8 \times 6$ nodes' SFE per the shortest wavelength is sufficient to simulate collinear wave mixing compared to twenty traditional finite elements.

(2) The proposed spectral finite elements are capable of increasing computational efficiency by as much as 14 times while maintaining the same accuracy in comparison with traditional FEM. Several numerical simulations with 15 million DOFs utilizing traditional FEM, 4.5 million DOFs utilizing $3 \times 3$ nodes' SFE, and 2.1 million DOFs utilizing $8 \times 6$ nodes' SFE have been proposed to prove this advantage for large-scale computations of collinear wave mixing, respectively.

(3) Implementing spectral finite element method using VUEL subroutine is exceedingly efficient when dealing with large-scale computations via multiple computing nodes.

(4) The proposed $8 \times 6$ nodes' SFE is recommended for collinear wave mixing to detect damage, as it can offer more accuracy with similar efficiency compared to 3 $\times 3$ nodes' SFE.

\section{Conflict of Interests}

The authors declare that there is no conflict of interests regarding the publication of this paper.

\section{Acknowledgments}

This work was supported by the National Natural Science Foundation of China under Grants 51378438 and 51509139; the National 973 Program under Grant 2013CB036204; the Postdoctoral Foundation of China under Grant 2015M570108; and the specific research funding from Ministry of Education under Grant SWJTU12ZT05.

\section{References}

[1] A. J. Croxford, P. D. Wilcox, B. W. Drinkwater, and P. B. Nagy, "The use of non-collinear mixing for nonlinear ultrasonic detection of plasticity and fatigue," The Journal of the Acoustical Society of America, vol. 126, no. 5, pp. EL117-EL122, 2009.

[2] A. Demčenko, L. Mainini, and V. A. Korneev, "A study of the noncollinear ultrasonic-wave-mixing technique under imperfect resonance conditions," Ultrasonics, vol. 57, pp. 179-189, 2015.

[3] M. Liu, G. Tang, L. J. Jacobs, and J. Qu, "Measuring acoustic nonlinearity parameter using collinear wave mixing," Journal of Applied Physics, vol. 112, no. 2, Article ID 024908, 2012.

[4] G. Tang, M. Liu, L. J. Jacobs, and J. Qu, "Detecting plastic strain distribution by a nonlinear wave mixing method," in Review of Progress in Quantitative Nondestructive Evaluation, vol. 32, American Institute of Physics, College Park, Md, USA, 2013.

[5] G. Tang, Wave Propagation in Nonlinear Media and Its Applications in Nondestructive Damage Assessment of Metallic Materials, Northwestern University, 2013.

[6] Z. Chen, G. Tang, Y. Zhao, L. J. Jacobs, and J. Qu, "Mixing of collinear plane wave pulses in elastic solids with quadratic nonlinearity," Journal of the Acoustical Society of America, vol. 136, no. 5, pp. 2389-2404, 2014.

[7] Y. Zhao, Z. Chen, P. Cao, and Y. Qiu, "Experiment and FEM study of one-way mixing of elastic waves with quadratic nonlinearity," NDT \& E International, vol. 72, pp. 33-40, 2015.

[8] T. Liszka and J. Orkisz, "The finite difference method at arbitrary irregular grids and its application in applied mechanics," Computers and Structures, vol. 11, no. 1-2, pp. 83-95, 1980.

[9] W. Hackbusch, Multi-Grid Methods and Applications, vol. 4, Springer, Berlin, Germany, 1985.

[10] O. C. Zienkiewicz and R. L. Taylor, The Finite Element Method: Solid Mechanics, vol. 2, Butterworth-Heinemann, 2000.

[11] J. N. Reddy, An Introduction to the Finite Element Method, vol. 2, McGraw-Hill, New York, NY, USA, 1993.

[12] Y. Cho and J. L. Rose, "A boundary element solution for a mode conversion study on the edge reflection of Lamb waves," Journal of the Acoustical Society of America, vol. 99, no. 4, pp. 2097-2109, 1996.

[13] P. K. Banerjee and R. Butterfield, Boundary Element Methods in Engineering Science, vol. 17, McGraw-Hill, London, UK, 1981.

[14] A. Bergamini and F. Biondini, "Finite strip modeling for optimal design of prestressed folded plate structures," Engineering Structures, vol. 26, no. 8, pp. 1043-1054, 2004.

[15] Y. K. Cheung and L. Tham, The Finite Strip Method, vol. 17, CRC Press, 1997.

[16] Y. Sohn and S. Krishnaswamy, "Mass spring lattice modeling of the scanning laser source technique," Ultrasonics, vol. 39, no. 8, pp. 543-551, 2002.

[17] H. Yim and Y. Sohn, "Numerical simulation and visualization of elastic waves using mass-spring lattice model," IEEE Transactions on Ultrasonics, Ferroelectrics, and Frequency Control, vol. 47, no. 3, pp. 549-558, 2000.

[18] P. P. Delsanto, T. Whitcombe, H. H. Chaskelis, and R. B. Mignogna, "Connection machine simulation of ultrasonic wave propagation in materials. I: the one-dimensional case," Wave Motion, vol. 16, no. 1, pp. 65-80, 1992. 
[19] B. C. Lee and W. J. Staszewski, "Modelling of Lamb waves for damage detection in metallic structures. Part I. Wave propagation," Smart Materials and Structures, vol. 12, no. 5, pp. 804-814, 2003.

[20] S. Ham and K.-J. Bathe, "A finite element method enriched for wave propagation problems," Computers \& Structures, vol. 9495, pp. 1-12, 2012.

[21] I. Babuška, F. Ihlenburg, T. Strouboulis, and S. K. Gangaraj, "A posteriori error estimation for finite element solutions of Helmholtz' equation. Part I: the quality of local indicators and estimators," International Journal for Numerical Methods in Engineering, vol. 40, no. 18, pp. 3443-3462, 1997.

[22] I. Babuška, F. Ihlenburg, T. Strouboulis, and S. K. Gangaraj, "A posteriori error estimation for finite element solutions of Helmholtz' equation. Part II. Estimation of the pollution error," International Journal for Numerical Methods in Engineering, vol. 40, no. 21, pp. 3883-3900, 1997.

[23] F. Moser, L. J. Jacobs, and J. Qu, "Modeling elastic wave propagation in waveguides with the finite element method," NDT \& $E$ International, vol. 32, no. 4, pp. 225-234, 1999.

[24] W. Ostachowicz, P. Kudela, M. Krawczuk, and A. Zak, Guided Waves in Structures for SHM: The Time-Domain Spectral Element Method, John Wiley \& Sons, 2011.

[25] A. T. Patera, "A spectral element method for fluid dynamics: laminar flow in a channel expansion," Journal of Computational Physics, vol. 54, no. 3, pp. 468-488, 1984.

[26] Y. C. Hu, K. Y. Sze, and Y. X. Zhou, "Stabilized plane and axisymmetric Lobatto finite element models," Computational Mechanics, vol. 56, no. 5, pp. 879-903, 2015.

[27] P. Kudela, M. Krawczuk, and W. Ostachowicz, "Wave propagation modelling in 1D structures using spectral finite elements," Journal of Sound and Vibration, vol. 300, no. 1-2, pp. 88-100, 2007.

[28] R. Sridhar, A. Chakraborty, and S. Gopalakrishnan, "Wave propagation analysis in anisotropic and inhomogeneous uncracked and cracked structures using pseudospectral finite element method," International Journal of Solids and Structures, vol. 43, no. 16, pp. 4997-5031, 2006.

[29] M. Palacz and M. Krawczuk, "Analysis of longitudinal wave propagation in a cracked rod by the spectral element method," Computers \& Structures, vol. 80, no. 24, pp. 1809-1816, 2002.

[30] M. Krawczuk, M. Palacz, and W. Ostachowicz, "The dynamic analysis of a cracked Timoshenko beam by the spectral element method," Journal of Sound and Vibration, vol. 264, no. 5, pp. 1139-1153, 2003.

[31] D. Roy Mahapatra and S. Gopalakrishnan, "A spectral finite element model for analysis of axial-flexural-shear coupled wave propagation in laminated composite beams," Composite Structures, vol. 59, no. 1, pp. 67-88, 2003.

[32] P. Kudela, A. Zak, M. Krawczuk, and W. Ostachowicz, "Modelling of wave propagation in composite plates using the time domain spectral element method," Journal of Sound and Vibration, vol. 302, no. 4-5, pp. 728-745, 2007.

[33] D. Komatitsch, R. Martin, J. Tromp, M. A. Taylor, and B. A. Wingate, "Wave propagation in 2-D elastic media using a spectral element method with triangles and quadrangles," Journal of Computational Acoustics, vol. 9, no. 2, pp. 703-718, 2001.

[34] C. S. Rekatsinas, C. V. Nastos, T. C. Theodosiou, and D. A. Saravanos, "A time-domain high-order spectral finite element for the simulation of symmetric and anti-symmetric guided waves in laminated composite strips," Wave Motion, vol. 53, pp. 1-19, 2015.
[35] J. M. Carcione, D. Kosloff, A. Behle, and G. Seriani, "Spectral scheme for wave propagation simulation in 3-D elasticanisotropic media," Geophysics, vol. 57, no. 12, pp. 1593-1607, 1992.

[36] G. Seriani, "3-D large-scale wave propagation modeling by spectral element method on Cray T3E multiprocessor," Computer Methods in Applied Mechanics and Engineering, vol. 164, no. 1-2, pp. 235-247, 1998.

[37] D. Komatitsch and J. Tromp, "Introduction to the spectral element method for three-dimensional seismic wave propagation," Geophysical Journal International, vol. 139, no. 3, pp. 806-822, 1999.

[38] J.-H. Lee, T. Xiao, and Q. H. Liu, "A 3-D spectral-element method using mixed-order curl conforming vector basis functions for electromagnetic fields," IEEE Transactions on Microwave Theory and Techniques, vol. 54, no. 1, pp. 437-444, 2006.

[39] H. Peng, G. Meng, and F. Li, "Modeling of wave propagation in plate structures using three-dimensional spectral element method for damage detection," Journal of Sound and Vibration, vol. 320, no. 4-5, pp. 942-954, 2009.

[40] M. F. Hamilton and D. T. Blackstock, Nonlinear Acoustics, vol. 237, Academic Press, San Diego, Calif, USA, 1998.

[41] D. D. Muir, One-sided ultrasonic determination of third order elastic constants using angle-beam acoustoelasticity measurements [Ph.D. thesis], Georgia Institute of Technology, Atlanta, Ga, USA, 2009. 


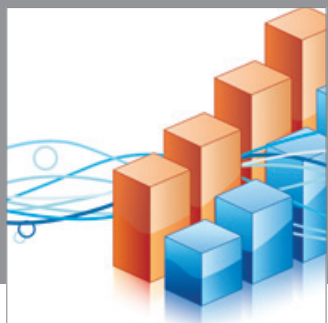

Advances in

Operations Research

mansans

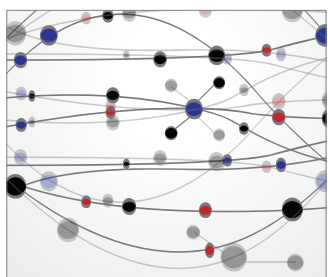

The Scientific World Journal
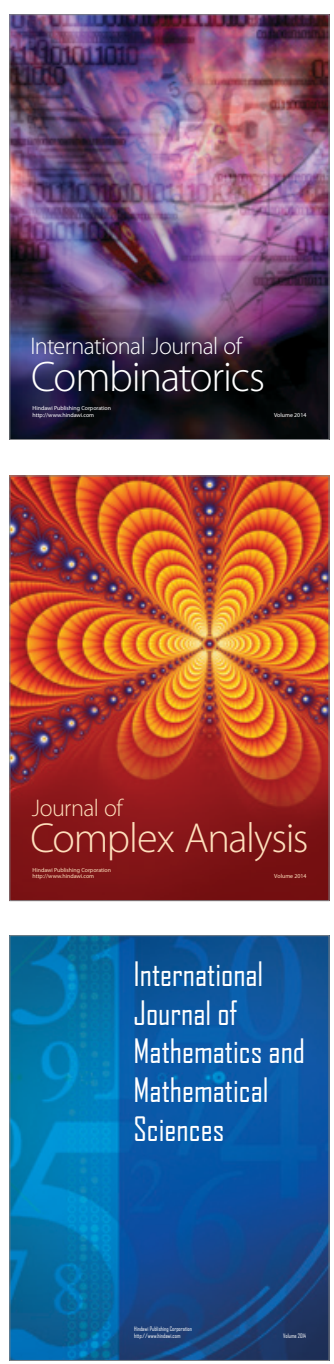
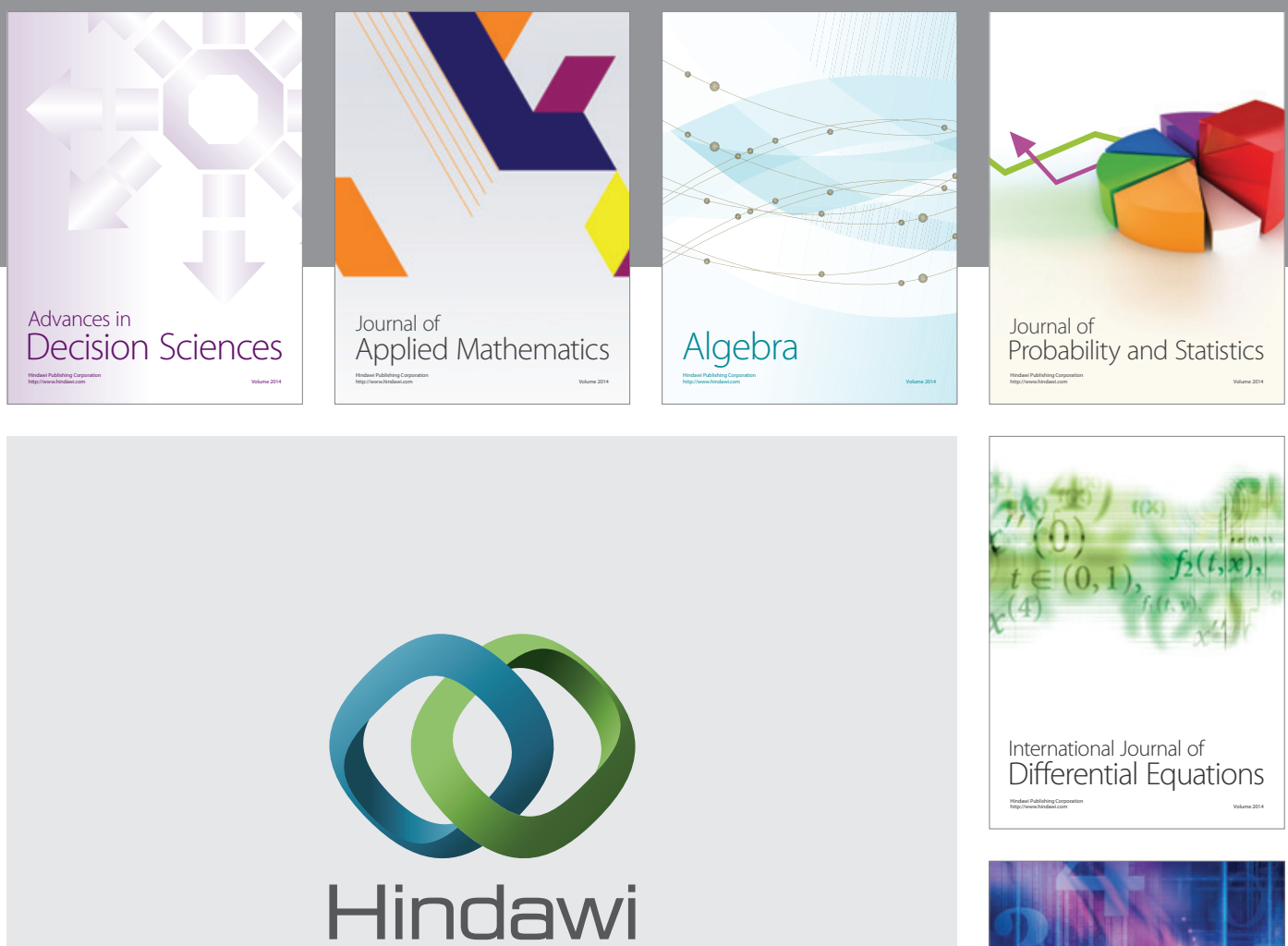

Submit your manuscripts at http://www.hindawi.com
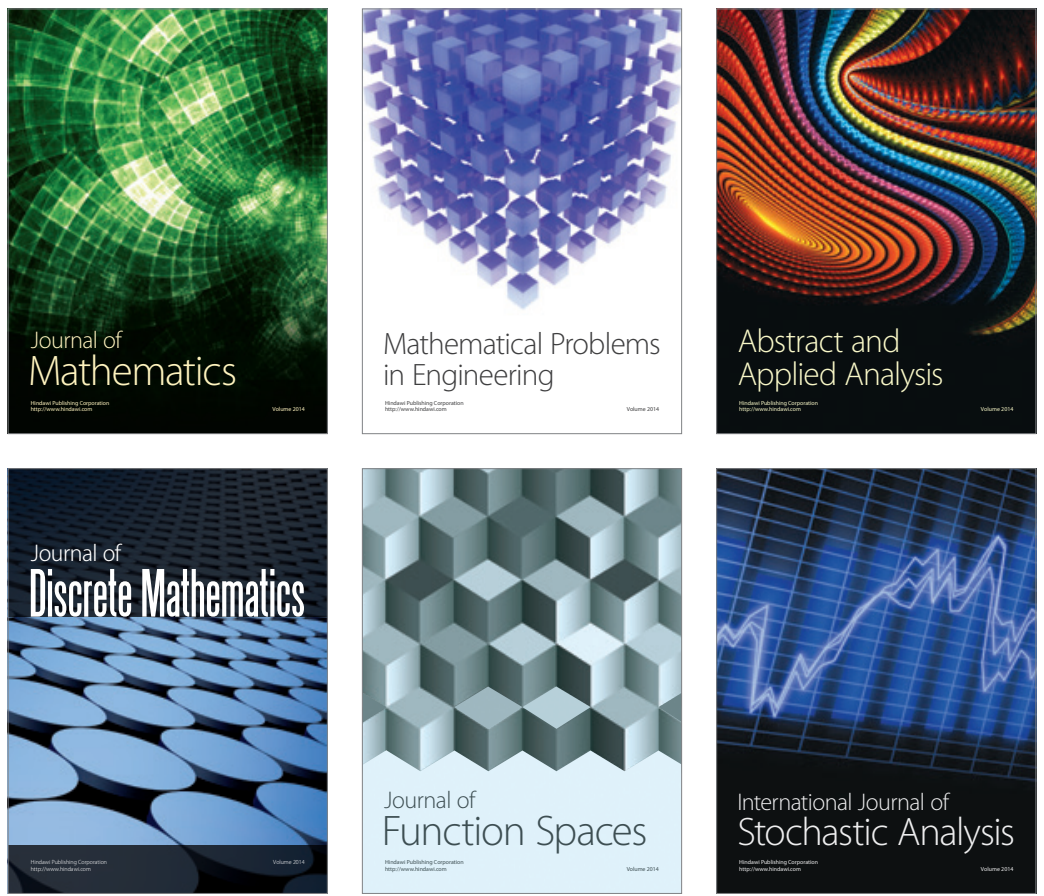

Journal of

Function Spaces

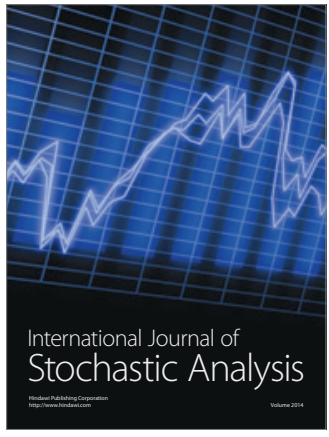

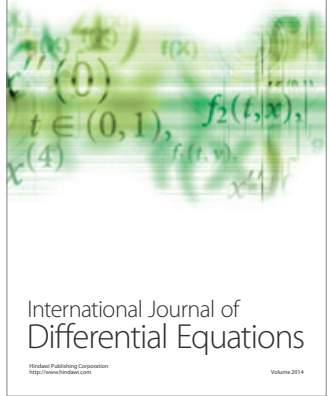
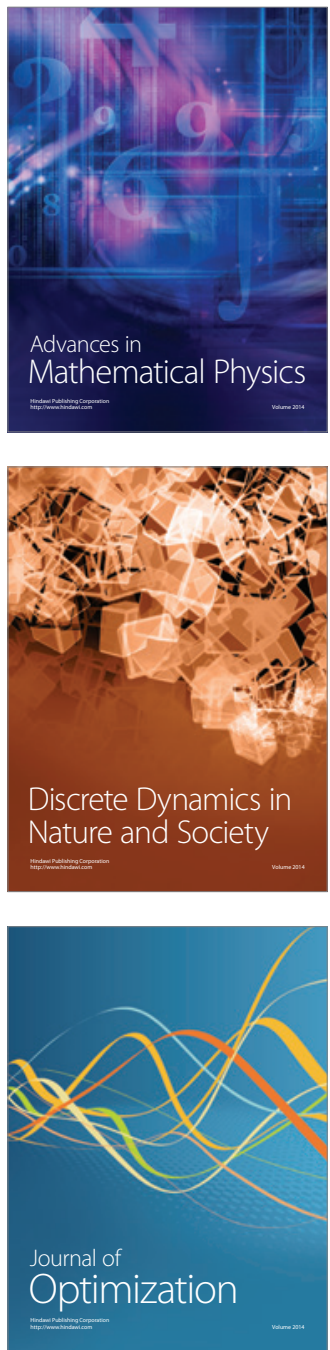